\title{
Cloning and characterization of a female gametophyte- specific gene in Gracilaria lemaneiformis (Gracilariales, Rhodophyte)
}

\author{
Peng Chen ${ }^{1,4}$, HongBo Shao ${ }^{1,2 \star}$ and $\mathrm{Di} \mathrm{Xu}^{3}$ \\ ${ }^{1}$ The CAS/Shandong Provincial, Key Laboratory of Coastal Environmental Processes, Yantai Institute of Costal Zone \\ Research, Chinese Academy of Sciences (CAS), Yantai 264003, China. \\ ${ }^{2}$ Institute for Life Sciences, Qingdao University of Science and Technology (QUST), Qingdao 266042, China. \\ ${ }^{3}$ College of Marine Life Sciences, Ocean University of China, Qingdao 266002, China. \\ ${ }^{4}$ Graduate University of the Chinese Academy of Sciences (CAS), Beijing 100049, China.
}

Accepted 30 May, 2011

\begin{abstract}
In this study, forward and reverse suppression subtractive hybridization (SSH) libraries were constructed between female and male gametophyte of Gracilaria lemaneiformis to isolate genes differentially expressed between gametophytes. Dot-blots were performed to screen 384 colonies randomly selected from each subtracted libraries. Partial gene (designated as GMF-01) which is female gametophyte-specific was selected to isolate its cDNA full length sequences and to be characterized. Then we got its cDNA full length sequences using SMART-RACE technic. Sequence analysis showed that the open reading frame (ORF) of GMF-01 is 1002 bp long with a GC content of $47.7 \%$, encoding 333 amino acids. GMF-01 does not have significant match in the databases when examined on NCBI website. Amino acid sequence analysis showed that the theoretical pl of this translated protein is 7.92 , while the instability index is computed to be 43.61 , which has classified the protein as unstable. Results of prediction showed $45.05 \%$ of its secondary structure has random coil and sub-cellular location prediction indicated that it is probably a extracellular protein.
\end{abstract}

Key words: Gracilaria lemaneiformis, suppression subtractive hybridization (SSH), sex determination, gametophyte.

\section{INTRODUCTION}

Gracilaria lemaneiformis is a commercially important agarophyte that can be used to produce agar, a major ingredient of dairy products, surgical jellies, ointments, cosmetics and healthcare products (Tseng, 2001). Gracilaria, along with Porphyra, Laminaria and Undaria has a bulk production by farming in China. It is not only an economically important algal species, but also a good material for genetical studies (Chen et al., 2009). Thus, it is important to pursue basic studies on $\mathrm{G}$. lemaneiformis.

Gracilaria has a life history involving three distinct stages: gametophytes, carposporophytes and

*Corresponding author. E-mail: shaohongbochu@126.com. Tel: 8653284023984. tetrasporophytes. Even though the phases and sexes of Gracilaria look identical before sexual maturation, there are physical differences between them (Kain and Destombe, 1995), such as growth rate in phases of $G$. lemaneiformis (Zhang and van der Meer, 1988), levels of polyamines in sexes and phases of G. cornea (GuzmanUriostegui et al., 2002), and lipid composition among different developmental stages of Gracilaria verrucosa (Khotimchenko, 2006). Owing to their particular life history, differentiation of phase and sex in red algae has already attracted researchers' attention. Researchers have been engaged in the study of the mechanisms of phase formation since 1976 (Ren and Zhang, 2008). However, no satisfactory results were obtained due to the limitation of applicable methods in the past. With the development of molecular biology, great progress has been made recently, such as that made by $\mathrm{Ye}$ et al. (2006). 6 ISSR primers, which had proved previously to 
be able to yield clear bands in Gracilaria, were used to distinguish the phases and sexes of $G$. lemaneiformis (Sun et al., 2003).

Until now, several phase-specific and sex-specific genes have been identified. Eight unique cDNAs for the sporophyte and seven specific for the gametophyte, including elongation factor alpha and lipoxygenase encoding genes have been isolated from Porphyra purpurea (Liu et al., 1996). A heat-shock protein encoding gene, which might be involved in the differentiation of female gametophyte, has been identified from Griffithsia japonica (Lee et al., 1998). An ubiquitin gene of G. lemaneiformis during phase formation is identified and characterized (Ren et al., 2009). GIRab11, the first functional Rab-like protein identified in G. lemaneiformis was isolated and the cDNA full-length of GIRab11 was obtained (Ren et al., 2008). cDNA subtracted hybridization was employed to study Porphyra purpurea phase-specific genes (Liu et al., 1994) and suppression subtractive hybridization (SSH) was deve-loped by Diatchenko et al. (1996), which turned out to be a successful tool for rapid screening of differentially expressed genes (Shim and Dunkle, 2002; De la Vega et al., 2007). SSH had been applied to study differential expression of genes in developmental stages (Brun et al., 2003; Zhu et al., 2003; Singh et al., 2007) and under stress conditions (Bahn et al., 2001; Caturla et al., 2002). Sun et al. (2002) reported an analysis of 180 ESTs of the G. lemaneiformis tetrasporophyte cDNA library. Suppression subtractive hybridization (SSH) was employed between RNA extracted from female gametophyte and tetrasporophyte. Fourteen cDNAs were identified, among which SSH466 was a putative tetrasporophyte-specific gene (Ren et al., 2006).

In this study, we constructed SSH libraries between female and male gametophyte of $G$. lemaneiformis and isolated the cDNA full-length of GMF-01, which is a female gametophyte-specific Gene.

\section{MATERIALS AND METHODS}

\section{Algae materials and cultivation}

G. lemaneiformis used in this study were collected from Zhanshan Bay (Qingdao, China). The healthy and mature fronds were used. Tetrasporophytes with released tetraspores were separated from female and male gametophytes under the microscope. Then one female gametophytes and one male gametophytes developed from tetraspores were picked out. The separated algae materials were brushed and rinsed in sterilized seawater until they were completely divorced from epiphytes. The materials were cultivated in Provasoli medium (Provasoli, 1966) under a light intensity of $50 \mu \mathrm{mol}$ photon $\mathrm{m}^{-2} \mathrm{~s}^{-1}$ with a $12: 12(\mathrm{~L}: \mathrm{D})$ cycle at $15 \pm 1^{\circ} \mathrm{C}$. The thallis were used for RNA extraction.

\section{SSH library construction}

To isolate sex-relative genes, suppression subtractive hybridization (SSH) was performed between RNA isolated from male and female gametophytes of $G$. lemaneiformis. Total RNA was extracted from each sample with RNeasy Plant Mini Kit (Qiagen, China), reversetranscribed and amplified using a SMART PCR cDNA Synthesis Kit (BD Biosciences Clontech, USA). Both forward (female gametophyte as tester and male gametophyte as driver) and backward (male gametophyte as tester and female gametophyte as driver) SSH were performed using a PCR-select CDNA subtraction kit (BD Biosciences Clontech) according to the manufacturer's instructions. In order to confirm differential expressions of the clones, cDNA dotblots were performed. Based on the results of cDNA dot-blots, clones that expressed differentially between female and male gametophyte were sequenced and aligned with the BLAST algorithms at the National Center for Biotechnology Information (NCBI). The sequences were also analysed with ContigExpress (Vector NTI Suite 6.0) to find contigs.

\section{Cloning of the cDNA full-length and sequence analysis of GMF-} 01

According to the partial sequence previously obtained and to get the full length of GMF-01, 5' and 3' CDNA ends were amplified with a BD SMART ${ }^{\mathrm{TM}}$ RACE CDNA amplification kit (BD Biosciences Clontech). Primers B5 (5'-GCGCTACCGTTGCTCCATAATCCAC3') and B3 (5'-CATCCAGTTCTACTCGCTTCTTATACC-3') were designed and respectively used in the $5^{\prime}$ and 3 ' RACE.

Sequence searches were performed by the BLAST algorithm on $\mathrm{NCBI}$ databases. Theoretical isoelectric point ( $\mathrm{pl}$ ) and molecular weight were predicted using ExPasy ProtParam tools (http://us.expasy. org/tools/protparam.html). Protein analysis was performed with ProtComp (www.softberry.com), TMHMM (http://www.cbs.dtu.dk/services/TMHMM-2.0/) and PSORT (http://psort.nibb.ac.jp/form2.html).

\section{RESULTS}

\section{Screening of the SSH cDNA libraries}

A total of 768 clones were randomly selected from the $\mathrm{SSH}$ libraries (384 clones from each of the two SSH libraries) and screened by macro-array dot-blots. Both forward and backward subtracted radioactively labeled cDNA populations were then used as individual probes for identical blots (Figure 1). 411 clones were found to be positive clones. When analysing the sequencing results with ContigExpress (Vector NTI Suite 6.0), 136 contigs were found and one female gametophyte-specific sequence (480 bp) showed significantly differential expression between the two sexes. It was designated as GMF-01 and was chosen to clone its full-length cDNA sequence. We are still analyzing the rest partial sequences and they will be published later. The partial sequence of GMF-01 is as follows:

GCAGACTTCTACTATTCAGTATAGATGGGTTGAAGAA TATAGGGCCACGTTTACCACCAAAGTGGAGATCGGA GAAATTATTCGGACGCAGGACATCATCAACTCGCCT GACTTTAACATGGGACAATCCGTTTCATTCGATGGAG TCGAGTGGAGTCCTCCAGTCAGCGACCGGAAGCCG CCGAACATTGGGGTAGCATACAAGGTTGACACGAAC GCTCTGCATCCAGTTCTACTCGCTTCTTATACCATGA 


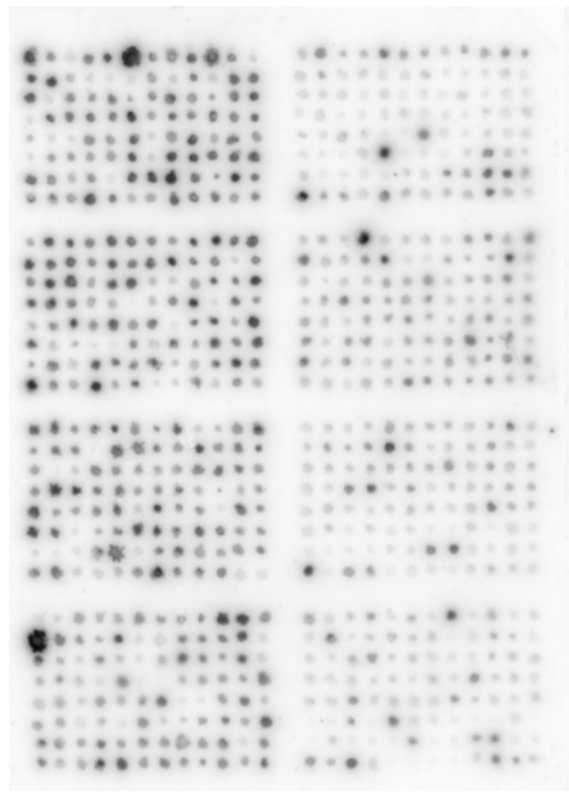

A

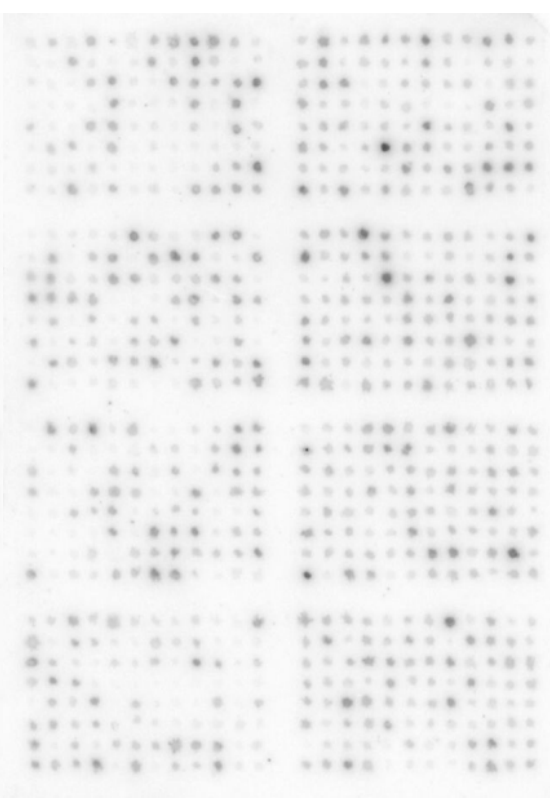

B

Figure 1. Dot blots of cDNA in subtractive. Two identical membranes blotted with PCR amplified CDNA sequences from subtracted libraries were probed with the forward subtracted probes (A) and the backward subtracted probes (B).

ACGGGCAAAAGAAATGGAGGCCGTTTTACTACGGCG TTTCTGTAATTTCTGGAAACACTTTGGTAGGCTTCCC CGTGATTAAGCTTCAGATCCAACTCGGTACTGCTATC AAGGATAGCGCATTCGTAGGAAGTATAATCGGCAAC AGTCTCGAAGTGGATTATGGAGCAACGGTAGCGCAA ACAGTGTGCGTGTACTCCCGTGAACGTGAGACCACG GTGAGGCT

\section{Cloning of the full-length cDNA and sequence analysis of GMF-01}

Based on the partial sequence obtained from the $\mathrm{SSH}$ library, two primers B5 and B3 were designed to amplify the 5' and 3' cDNA ends of GMF-01. 1019 bp and $548 \mathrm{bp}$ were amplified in the 5' and 3' SMART RACE reactions respectively. The full length of GMF-01 cDNA had 1357 nucleotides. Sequence analysis showed that the open reading frame (ORF) of GMF-01 is 1002 bp long with a GC content of $47.7 \%$, encoding 333 amino acids. Searches from the public sequence databases using $\mathrm{NCBI}$ BLASTx showed that there was no significant match with GMF-01. The predicted protein had a calculated molecular weight of $36.7 \mathrm{kDa}$ and a theoretical pl of 7.92.

The instability index was computed to be 43.61 which classified the protein as unstable. The GRAVY (Grand average of hydropathicity) of this protein was -0.131 , which indicated that the protein was hydrophilic. Results of prediction showed $45.05 \%$ of its secondary structure is random coil (Figure 2). Sub-cellular location prediction results with ProtComp, TMHMM (Figure 3) and PSORT all indicated that it's probably an extracellular protein. The cDNA and amino acid sequences are indicated in Figures 2 and 3.

\section{DISCUSSION}

As an agarophyte, components of $G$. lemaneiformis cells are extremely complicated. In the construction of $\mathrm{SSH}$ library, RNA isolated from $G$. lemaneiformis was too difficult to purify enough due to the polysaccharides. The SMART approach is a PCR-based amplification system that allows the creation of cDNA from a very small amount of total RNA (Cramer and Lawrence, 2004; Shary and Guha-Mukherjee, 2004; Pavan, 2011).

Thus, SMART strategy was taken before SSH library construction was carried out. That is the key point of the successfully construction of SSH library. We identified 3 practical sequences that are differentially expressed between female gametophytes and male gametophytes. One putative female gametophyte-specific gene GMF-01 was selected to isolate the full-length of cDNA for further analysis.

In this study, we identified a female gametophytespecific gene of $G$. lemaneiformis. The protein encoded by GMF-01 may be a extracellular protein. It was not known yet whether GMF-01 was red-algae specific.

In the SSH library, we found some special gene 


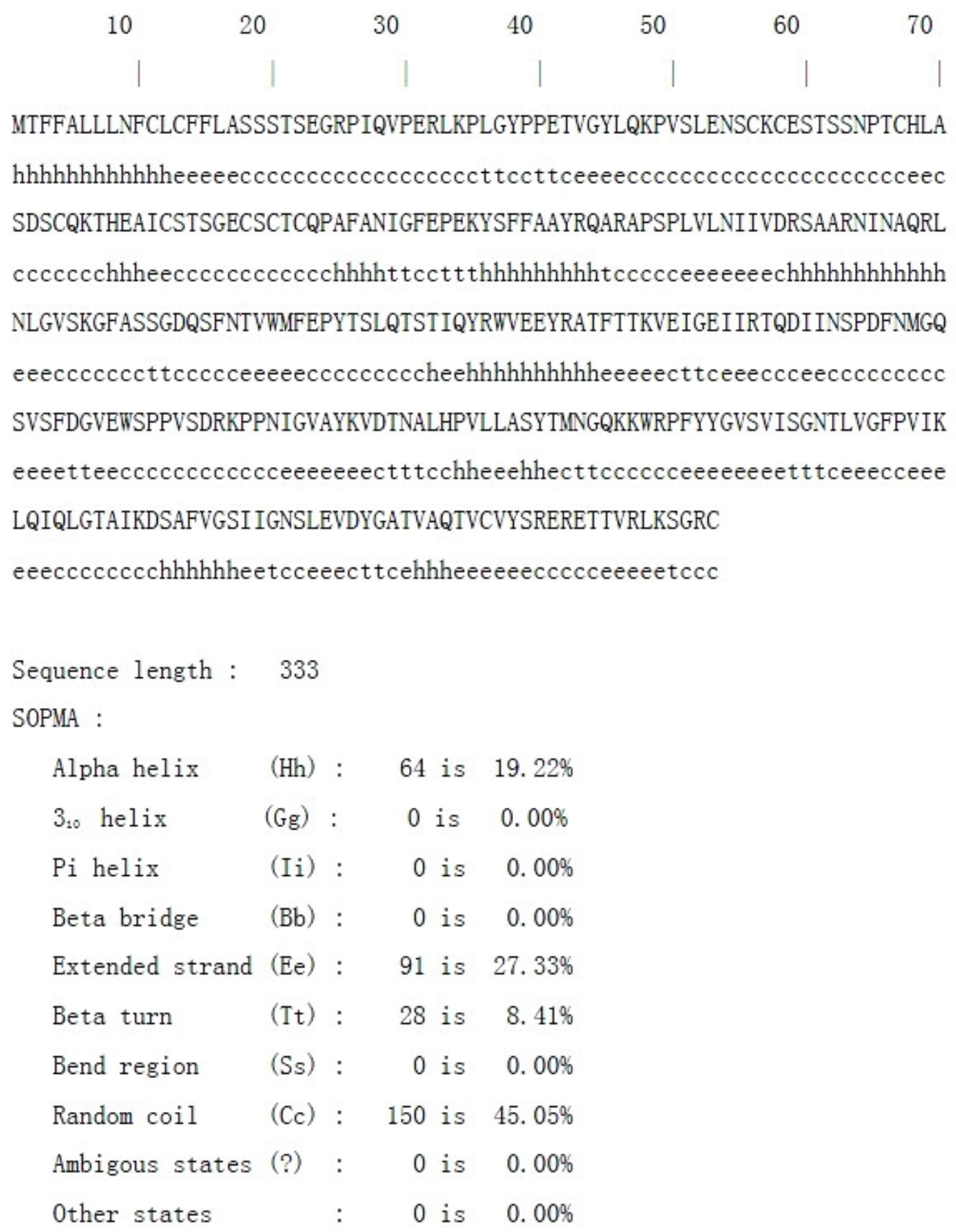

Figure 2. Prediction of secondary structure of GMF-01.

expression: (1) expression of rDNA and ribosomal protein, such as $16 \mathrm{SrDNA}$, 23SrDNA, ribosomal protein S8, L23, L27. This may indicate that a number of genes are synthesized during sex differentiation, (2) expression of $\alpha-1,4-g l u c a n$ lyase (Agll) which could degrade the floridean starch into glucose which can provide energy for sex differentiation, (3) expression of some protein genes related to cellular process, including aminotransferase, COP9 signalosome, Ran (one of GTP-binding proteins).
These proteins play important roles in cell division and signal transduction. However, their function in sex regulation needs additional research.

The results in this study strongly suggest that GMF-01 is a functional gene that may play important roles during the sex determination of $G$. lemaneiformis. We are now committed to further studies of GMF-01, such as gene expression analysis and semi quantitative determination by RT-PCR, more work are required to elucidate the 
TMHMM posterior probabilities for GMF-01

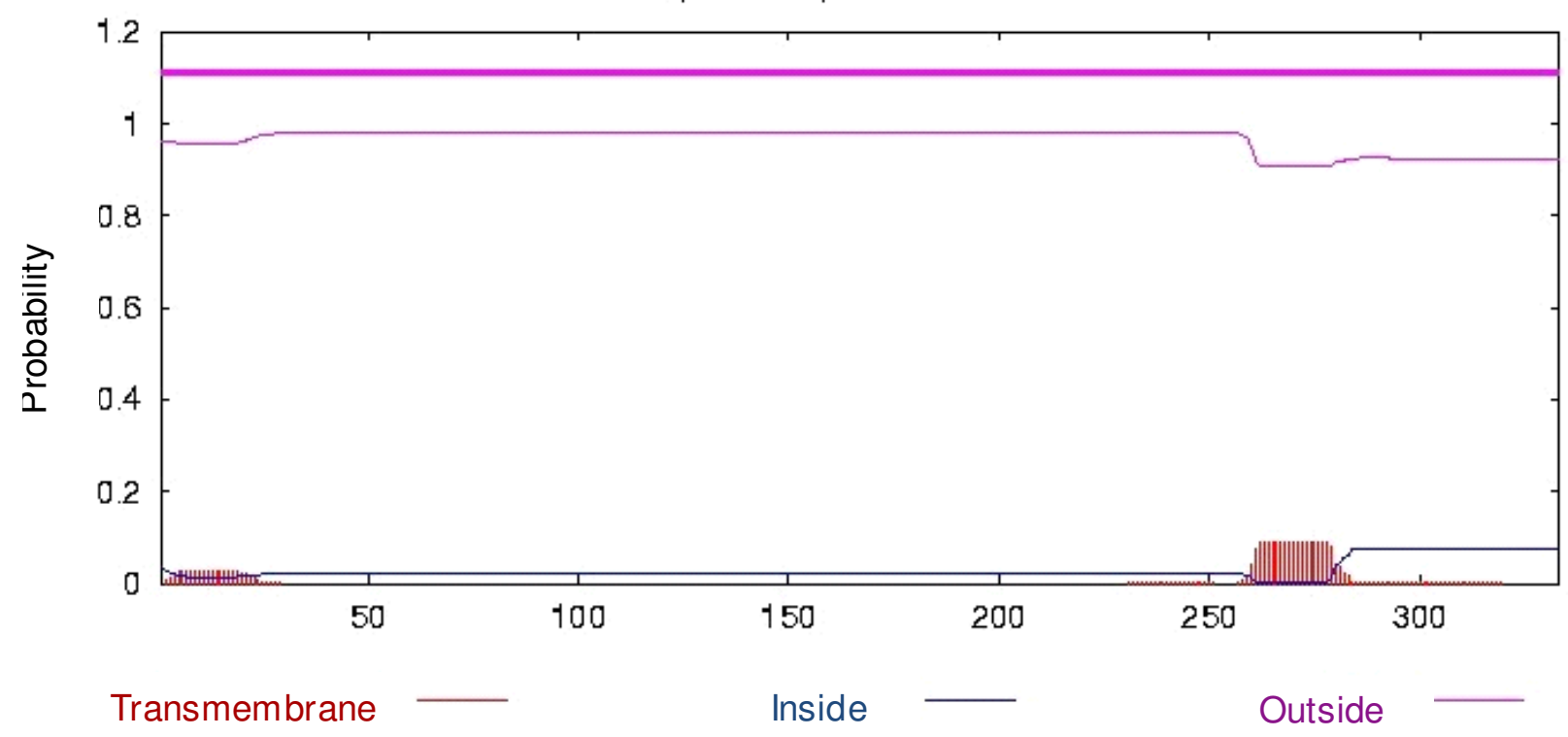

Figure 3. TMHMM posterior probabilities for GMF-01.

function of GMF-01. The construction of additional transgenic clones in which GMF-01 is knocked out should allow a better assignment of its function. Based on the SSH libraries constructed in this study, more differentially expressed genes could be found. The differentially expressed genes obtained in this study are closely related with gametogenesis of $G$. lemaneiformis. Studies on these genes may play important roles in understanding sex determination mechanisms and will provide clues for red algal evolution pathways.

\section{ACKNOWLEDGEMENTS}

This work was jointly supported by National Scientific Foundations of China (No. 40606034),

\section{REFERENCES}

Bahn SC, Bae MS, Park YB, Oh SI, Jeung JU, Bae JM (2001). Molecular cloning and characterization of a novel low temperatureinduced gene, blti2, from barley (Hordeum vulgare L). Biochem. Biophys. Acta., 1532: 134-137.

Brun F, Gonneau M, Laloue M, Nogue F(2003). Identification of Physcomitrella patens genes specific of bud and gametophore formation. Plant Sci., 165: 1267-1274.

Caturla M, Chaparro C, Schroeyers K, Holsters M (2002). Suppression subtractive hybridization to enrich low-abundance and submergenceenhanced transcripts of adventitious root primordia of Sesbania rostrata. Plant Sci., 162: 915-921.

Chen P, Shao HB, Xu D, Qin S (2009).Progress in Gracilaria Biology and Developmental Utilization: Main Issues and Prospective. Rev. Fish Sci., 17:494-504.

Cramer RA, Lawrence CB (2004). Identification of Alternaria brassicicola genes expressed in planta during pathogenesis of Arabidopsis thaliana. Fungal Genet. Biol., 41: 115-128.

De la Vega, E, Degnan BM, Hall MR, Wilson KJ (2007). Differential expression of immune-related genes and transposable elements in black tiger shrimp (Penaeus monodon) exposed to a range of environmental stressors. Fish Shellfish Immunol., 23: 1072-1088.

Diatchenko L, Lau YC, Campbell AP, Chenchik A, Moqadam F, Huang B (1996). Suppression subtractive hybridization: a method for generating differentially regulated or tissue-specific CDNA probes and libraries. Proc. Natl. Acad. Sci., USA., 93: 6025-6030

Guzman-Uriostegui A, Garcia-Jimenez P, Marian F, Robledo D, Robaina $R$ (2002). Polyamines influence maturation in reproductive structures of Gracilaria cornea (Gracilariales, Rhodophyta). J. Phycol., 38: 1169-1175

Kain JM, Destombe C (1995).A review of the life history, reproduction and phenology of Gracilaria. J. Appl. Phycol., 7: 269-281

Khotimchenko SV (2006). Variations in lipid composition among different developmental stages of Gracilaria verrucosa (Rhodophyta). Bot. Mar., 49:34-38

Lee YK, Kim SH, Hong CB, Chah OK, Kim GH (1998). Heat-shock protein 90 may be involved in differentiation of the female gametophytes in Griffithsia japonica (Ceramiales, Rhodophyta). J. Phycol., 34: 1017-1023.

Liu QY, Baldauf SL, Reith ME (1996). Elongation factor 1 alpha genes of red alga Porphyra purpurea include a novel, developmentally specialized variant. Plant Mol. Biol., 31: 77-85.

Liu QY, van der Meer JP, Reith ME (1994). Isolation and characterization of phase-specific complementary DNAs from sporophytes and gametophytes of Porphyra purpurea (Rhodophyta) using subtracted complementary DNA libraries. J. Phycol., 30: 513520.

Pavan KP (2011). Influence of five industrial pollutants on mycorrhizal colonization, nodulation and growth of Acacia nilotica and Leucaena leucocephala. Afr. J. Microbiol. Res., 5: 93-95.

Povasoli L (1966). Media and prospects of the cultivation of marine algae. In: Watanabe A, Hattori A (eds) Cultures and collections of algae. Proceedings of the US-Japan Conference Hakone, Japan, September 1966. Japanese Society of Plant Physiology, Tokyo, pp. $1-26$

Ren XY, Sui ZH, Mao YX, Zang XN, Xu D, Zhang XC (2009). Cloning and characterization of two types of ubiquitin genes from Gracilariopsis lemaneiformis (Gracilariales, Rhodophyta). J. Appl. Phycol., 21: 273-278.

Ren XY, Sui ZH, Zhang XC (2006). Cloning and Characterization of Glyceraldehyde-3-phosphate Dehydrogenase Encoding Gene in 
Gracilaria /Gracilariopsis lemaneiformis. J. Ocean Univ. Chin., 2: 146150.

Ren XY, Zhang XC (2008).Identification of a Putative TetrasporophyteSpecific Gene in Gracilaria lemaneiformis (Gracilariales, Rhodophyte). J. Ocean Univ. Chin., 3: 299-303.

Ren XY, Zhang XC, Mao YX, Sui ZH, Xu D, Zang XN (2008). Cloning and characterization of a Rab11 homologue in Gracilariopsis lemaneiformis. J. Appl. Phycol., 20: 1103-1109

Shary S, Guha-Mukherjee S (2004).Isolation and expression studies of differentiation-specific genes in tobacco dihaploids using PCR-based subtractive hybridization method. Plant Sci., 166: 317-322.

Shim WB, Dunkle LD (2002). Identification of genes expressed during cercosporin biosynthesis in Cercospora zeae-maydis. Physiol. Mol. Plant Pathol., 61: 237-248.

Singh M, Burson BL, Finlayson SA (2007).Isolation of candidate genes for apomictic development in buffelgrass (Pennisetum ciliare). Plant Mol. Biol., 64: 673-682
Sun X, Yang GP, Mao YX, Zhang XC, Sui ZH, Qin S (2002) Analysis of expressed sequence tags of a marine algae, Gracilaria/Gracilariopsis lemaneiformis. Progr. Nat. Sci., 12: 518-523.

Sun X, Zhang XC, Mao YX, Liu JJ, Sui ZH (2003). ISSR analysis of marine red algae Gracilaria (Rhodophyceae). High Technol. Lett., 13: 89-93.

Tseng CK (2001).Algal biotechnology industries and research activities in China. J. Appl. Phycol., 13: 375-380.

Ye NH, Wang HX, Wang QC (2006).Formation and early development of tetraspores of Gracilaria lemaneiformis (Gracilaria, Gracilariaceae) under laboratory conditions. Aquaculture 254: 219-226.

Zhang XC, van der Meer JP (1988) A genetic study on Gracilaria sjoestedtii. Can. J. Bot., 66: 2022-2026.

Zhu Y, Johnson TJ, Myers AA, Kanost MR (2003). Identification by subtractive suppression hybridization of bacteria induced genes expressed in Manduca sexta fat body. Insect Biochem. Mol. Biol., 33: 541-559 